\title{
IUD fitters and training in resuscitation
}

I have been reading the correspondence on the subject of intrauterine device/system (IUD/IUS) fitters and training in resuscitation in the Journal with interest. ${ }^{1-3}$

It seems that IUD/IUS fitting has now been suspended in many clinics where there is not a doctor on the premises able to administer an intravenous injection of atropine. I have been fitting IUDs for over 30 years, often fitting more than 100 a year. I have never once been in a situation where I have even considered administering atropine. When there has been a situation where the patient has experienced vagal shock, then basic life support measures have been all that is required. Nurses can do this just as well as doctors.

In Lincolnshire we have developed many of our clinics as nurse-led as it is increasingly difficult to find doctors to staff rural clinics when they are able to earn very much more doing sessions in general practice. I think that this is a situation that is likely to worsen as the present generation of clinic doctors retire from the service.

It is important that family planning clinics are able to offer all forms of contraception, and they may be the only setting where a patient can have an IUD as emergency contraception. It is a tragedy if well-trained, experienced nurses are being prevented from offering a service that they are very capable of giving because of non-evidence-based restrictions.

It is to be hoped that the Royal College of Nursing and the Faculty of Sexual and Reproductive Healthcare can get together and clarify this situation, and that nurses can again be able to practise their skills in intrauterine insertions.

Beth Devonald, MRCGP, DFSRH

21 Blind Lane, Coleby, Lincoln LN5 OAL, UK; devonald@btinternet.com

Competing interests None.

J Fam Plann Reprod Health Care 2011;37:61. doi:10.1136/jprhc.2010.0028

\section{REFERENCES}

1 Hollingworth B. Need for IUD fitters to have expertise in resuscitation [Letter]. J Fam Plann Reprod Health Care 2010;36:179-180.

2 Mansour D. Nurse training and the need for IUD fitters to have expertise in resuscitation [Letter]. J Fam Plann Reprod Health Care 2010;36:180.

3 Mehigan S. Reply [Letter]. J Fam Plann Reprod Health Care 2010;36:180. 